\title{
Not a duty but an opportunity: exploring the lived experiences of community health workers in Indiana through photovoice
}

\author{
Ryan I. Logan \\ Department of Anthropology, University of South Florida, Tampa, Florida, USA
}

\begin{abstract}
Few studies have conducted photovoice projects to explore themes related to the lived experiences of community health workers (CHWs). As a relatively unknown segment of the health care workforce in the United States, CHWs are typically members of the communities they work within and fulfill unique and complementary roles through health education, health prevention, community outreach, and advocacy. This article documents a photovoice project that assessed the strengths, challenges, impacts, and what it means to be a CHW in Indiana. In this project, CHWs participated in the method of photovoice in two formal meetings - one to introduce the method and another to analyze the photographs. Participants displayed photographs, interpreted these images, and co-constructed their lived experiences. Several key themes emerged from discussion of the photographs including: participant descriptions of building individual and character traits, conceptualizations of this position, and how participants connect disparate resources for their clients. Themes and findings from this research can be utilized to reach out to potential employers and policy makers regarding the integration of CHWs into the workforce. Overall, this project documents the lived experiences of CHWs and highlights their voices as they become an accepted member within the broader workforce.
\end{abstract}

\section{Introduction}

In this article, I detail a photovoice project that explored the lived experiences of community health workers (CHWs) operating primarily in Indianapolis, Indiana. Despite being present in this state for several decades and comprising an estimated 670-1,260 workers, ${ }^{1} \mathrm{CHWs}$ are relatively unknown by medical professionals and the general public. This lack of awareness complicates their in-

Correspondence: Ryan I. Logan, Department of Anthropology, University of South Florida, 4202 E. Fowler Avenue, SOC100, Tampa, Florida, 33620, USA.

Tel.: +1.813.974.2138.

E-mail: ryanlogan@mail.usf.edu

Key words: Community health workers; Photovoice; Access to care; Health disparities; Lived experience; Indiana.

Conflict of interest: the author declares no potential conflict of interest.

Funding: none.

Received for publication: 10 September 2018.

Revision received: 19 November 2018.

Accepted for publication: 19 November 2018.

This work is licensed under a Creative Commons Attribution NonCommercial 4.0 License (CC BY-NC 4.0).

C Copyright R.I. Logan, 2018

Licensee PAGEPress, Italy

Qualitative Research in Medicine \& Healthcare 2018; 2:132-144 doi:10.4081/qrmh.2018.7816 tegration within the broader workforce and, thereby, fully realizing their unique roles that can be harnessed to improve the health outcomes of marginalized populations. ${ }^{2,3}$ Moreover, this state-level lack of awareness also resonates on national scale and among other high-income countries. ${ }^{4}$ In spite of this, there are an estimated 54,760 CHWs operating in the U.S. alone. ${ }^{1}$ Given the diversity, breadth, and potential for improved health outcomes, further understanding is needed regarding the nuanced issues encountered by CHWs at the ground level as well as amplifying the voices of these workers through collaborative methods such as photovoice.

As noted by the American Public Health Association, CHWs fulfill a wide range of unique services that can improve health and social issues outside the realm of the clinic including community outreach, health education/prevention, and advocacy. Due to their ability to address wide gaps in health care coverage, especially through addressing social determinants of health, CHWs are poised to make significant contributions to the U.S. health care system. ${ }^{5,6}$ In spite of these unique and complementary functions, CHWs remain largely on the fringes of the health care workforce in Indiana and throughout much of the United States. ${ }^{7}$ As a result, additional studies are needed that elucidate the lived experiences of this workforce - including how they conceptualize their position, specific challenges encountered, and the impacts they make in their communities. These lived experiences provide crucial theoretical insights but also applied implications - especially in terms of informing policy development that seeks to integrate CHWs into the broader workforce.

Photovoice is a participatory methodology that pro- 
duces visual data regarding a particular issue or topic that are collected, interpreted, and analyzed by participants. ${ }^{8,9}$ This method is particularly compelling when used in conjunction with CHWs due to the intimate knowledge they possess of their communities. This method also provides a means to reconstruct the concept of community through participant interpretation. As a result, participants reflexively address the notion of the community and thereby demonstrate that it is not a monolithic and static construct but rather a dynamic and contested space. Several scholars have suggested engaging CHWs as research partners, ${ }^{10-12}$ while others have specifically utilized photovoice to explore pertinent grassroots-level issues among these workers. ${ }^{13-15}$ Photovoice served as a lens into the lives of these workers that also enhanced the participants' role within the project. CHWs took photographs in response to three prompts and, additionally, co-constructed their interpretations and analyses through group discussion. In this way, photovoice facilitates an active role for participants during the course of the project and analysis of the findings. This method also provides provided a crucial environment in which participants interpreted their images and elevated their voices within the project. This aspect of photovoice was crucial given that data collection traditionally extracts data to be analyzed by the researcher, with little additional input from participants.

I conducted this photovoice project in part as a collaborative effort with a small yet burgeoning CHW organization. The photovoice project served as one of the methods I utilized in my overarching dissertation project. Aside from gaining a deeper understanding of the lived experiences of these participants, this project was timely given that the state government of Indiana had recently approved a CHW certification and had convened a workgroup of stakeholders to develop legislation regarding this position. As a result, gaining further insights into the lived experiences of these workers was crucial - especially as steps are being taken to develop this position into a more formal component of the health and social services workforce. Aside from producing novel, participant-interpreted findings, photovoice also functioned as a method that fosters applied implications through fomenting critical consciousness - especially through the dissemination of these images to raise awareness on the part of the policy makers, medical professionals, and the public.

\section{Background}

In spite of a general lack of awareness, CHWs have been quantitatively demonstrated to improve a variety of health outcomes among marginalized populations. ${ }^{16}$ These workers have also served as advocates for social justice essentially since their inception. ${ }^{12}$ Although their usage waxed and waned throughout the subsequent decades in the U.S., CHWs were mentioned by name in the Patient Protection and Affordable Care Act of 2010 (ACA). The ACA outlined several potential roles for CHWs and also acknowledged them as a new member of the health care team (that includes doctors, nurse practitioners, nurses, and other established professions). ${ }^{3}$ This national level recognition was vital in highlighting the role and unique contribution of the CHW model.

However, due to a lack of a national policy and framework dedicated setting guidelines and rules regarding the CHW model, state governments and various employing organizations have lead the way in terms developing and implementing this position. As a result, CHWs are utilized at varying capacities and, often, under differing terminologies. Several states, such as Massachusetts, Minnesota and Oregon, have well-established CHW programs. ${ }^{3,7}$ Minnesota is particularly unique due to its extensive listing of CHW-specific services that are reimbursable through Medicaid. ${ }^{3}$ Due to these state-by-state variations, their utilization, terminology, specific functions, and experiences at the grassroots level varies greatly. While national recognition in the form of the ACA and various state-level policies have sought to integrate CHWs, they have largely continued to exist on the fringes of the workforce.

CHW programs faced criticism in the 1980 s following the failure of some programs to achieve their desired outcomes. Studies reported mixed results related to the outcomes of CHW programs and that political and financial factors further negatively impacted them. ${ }^{7}$ As a result, these issues largely caused the downfall of this model in addition to widespread ambivalence regarding its broader adoption. However, public health studies have demonstrated the quantitative impact of these workers on a variety of health outcomes - particularly chronic disease prevention and management. ${ }^{3,16}$ Furthermore, some public health studies have reported CHW programs to be costeffective. ${ }^{16-18}$ Thus, despite mixed results previously experienced by CHW programs in the U.S., current public health evidence demonstrates their potential for positive health outcomes and cost-effectiveness. ${ }^{3,16,18}$

Although photovoice is a well-established methodology, few studies have utilized this method to explore various topics related to CHWs. Mayfield-Johnson et al. ${ }^{14}$ conducted a photovoice project with community health advisors (CHAs) in order to assess changes in feelings of empowerment. Their study revealed critical insights into several key themes explored by CHAs and provided crucial data in further utilizing CHAs in the workforce. Baquero et al. ${ }^{13}$ discussed the results of using CHWs to reduce barriers to reproductive and sexual health care. The authors also described how the photographs were used in a community forum to discuss and validate the findings. Finally, Mitchell et al. ${ }^{15}$ drew on photovoice to explore HIV/AIDS in South Africa and also as a reflexive method to create a plan of action. In these examples, photovoice served as a versatile methodology that not only enhance the role and voice of the participants but also yielded critical visual data.

Indiana has lagged behind other states in terms of de- 
veloping and utilizing this workforce. In spite of this, CHWs have a long history in the state and are employed in various capacities in both health and social service organizations. Some CHWs have as much as 30 years of experience working either as paid and/or as volunteers and have been employed in clinics, hospitals, and/or social service organizations. However, those that are hired are normally paid through short-term, grant-funded positions and thus maintain tenuous employment. This soft funding of CHWs often results in the position ending after a short period of time and thereby keeps them from formal integration within the broader workforce. ${ }^{7}$ Additionally, there currently are over 60 titles in use which fit underneath the CHW umbrella within the state. This myriad of titles creates confusion over their roles and responsibilities in addition to presenting challenges for crafting policy regarding this workforce. The plethora of titles also contributes to the lack of awareness on the part of medical professionals and the public about who are CHWs and what, exactly, it is that they do.

However, there are crucial steps being taken at the grassroots and government levels in Indiana. A statewide CHW organization formed in 2015 to advocate for the development of this workforce and has made connections with other organizations and the state government. At the time of the photovoice project in 2017, the governor had convened a workgroup to develop policy regarding the CHW model. The workgroup drafted and provided recommendations for an official model as well as a set of CHWspecific, Medicaid reimbursable services. While these are important steps toward legitimizing these workers, the lack of awareness regarding CHWs, fully utilizing their unique skillset once employed, and ground level challenges persist as lingering issues that hinder their integration. Overall, this project served to illuminate the lived experiences of CHWs - through their active participation in a photovoice project - in addition to producing findings that will raise awareness of this workforce as they gain further acceptance into health and social services organizations.

\section{Photovoice: method and theoretical orientation}

Photovoice is a participatory methodology that produces visual data - in the form of photographs - and also elevates the involvement of the research participants. ${ }^{8,9,19}$ Specifically, the participants take photographs in relation to a prompt or theme and are involved in the production, selection, interpretation, and analysis of the photographs. ${ }^{8,9,19,20}$ The analysis phase is particularly important as participants explain and interpret their photographs as well as co-construct meaning through group discussion. As a result of this enhanced involvement, research participants can address issues of representation in photographs through the inclusion of their own interpretations. Photovoice has several strengths that set it aside from other forms of qualitative research methods. Wang and Burris (p. 369) ${ }^{9}$ describe three crucial strengths as i) empowering community members to record and reflect on their community's strengths and needs, ii) fostering discussion among the community members about topics stemming from the photographs, and iii) as a means to reach policy makers.

Additionally, the theoretical orientation of this method fosters discussions that can yield empowerment, action, and social justice regarding the topic of the project. ${ }^{14,20}$ Photovoice is grounded in feminist theory, empowerment education, and critical consciousness. ${ }^{8,14,19,20}$ These theoretical foci are crucial in framing the method and participants toward fostering critical consciousness that can be operationalized into action. Feminist theory provides a framing that emphasizes the lived experience and interpretation from the point of view of the participant, thereby elevating the voice of the participant within the research. This orientation is particularly vital as photovoice provides a lens to showcase the positionality and personal experience of the participant. Photovoice also provides an environment that facilitates the co-construction of knowledge through discussions of individual interpretations contrasted with others' experiences. In this way, photovoice draws on Paulo Freire's critical consciousness through empowerment education, in which teachers and students co-create knowledge. . $^{14,1920}$ Through photographs, interpretations, discussions, and the co-construction of knowledge, participants critically analyze social and structural issues related to their experiences. In fostering critical consciousness, participants can foment potential for producing positive social change. ${ }^{14,20}$ As a result, photovoice serves as a method that collaboratively engages participants, elevates their voice in the research, and can foster policy change and social justice.

\section{Materials and Methods}

\section{Study design}

Photovoice served as a methodological complement to the collaborative anthropology approach that framed the overarching dissertation project. Collaborative anthropology, as defined by Bade and Martinez,${ }^{21}$ is an approach that emphasizes developing a long-term and committed relationship with the community partner (e.g., participants, stakeholders) in which the needs of all parties are expressed including that of the partner(s) and researcher(s). In this way, the researcher(s) and participants identify the primary issues to explore in the project and that all parties are involved in the analysis and writeup. Like other participatory action research methodologies, collaborative anthropology strives to remove the traditional power dynamic between researcher and participants. A more egalitarian relationship is developed between all parties involved due to the enhanced involvement of participants in the design, process, analysis, and write-up. As a result, photovoice pairs well with 
this collaborative framework due to its theoretical foundations in feminist theory and empowerment education. ${ }^{9,14,19,20}$

In order to carry out the photovoice project, I worked with the CHW organization's leadership to collaboratively develop a set of three prompts. These prompts provided critical information regarding the meaning, impacts, and challenges that shape the lived experience of CHWs in Indiana. The formation of the project began in the summer of 2017 and recruitment occurred in August and September. Participants attended the first photovoice meeting in October and concluded in a final meeting in December. The University of South Florida Institutional Review Board (USF IRB) approved this project. Per USF IRB guidelines, all participants signed an informed consent document as well as a copyright release granting permission for use of their photographs.

\section{Recruitment and sampling}

Although eight CHWs were recruited for the project, two were lost to follow-up after the initial meeting. Six participants in total completed the project from start to finish (Table 1). The recruited participants were from a variety of different organizations. Thus time commitment to the project was challenging for many and was the primary reason for the participants lost to follow-up. Those recruited for the project were from interested participants from the overarching dissertation project and via wordof-mouth. CHWs who expressed interest were given a brief explanation of photovoice, its aims, and the overarching project prior to the first official meeting. Participants who were still interested were then provided with the date and time of the first formal meeting to discuss the photovoice project.

Due to the busy schedules of the participants, two formal meetings were held at the partner organization. The first meeting lasted an hour and a half and fully introduced the concept of photovoice, demonstrated several different examples of photovoice projects, discussed ethics and representation in photography, basic photography tips, and introduced the photovoice prompts. As participants utilized their smartphones for the project, they were also given a handout that detailed smartphone camera tips. Due to USF IRB guidelines, participants were unable to take photographs that contained identifiable individuals. And, as CHWs operate within the guidelines of the Health Insurance Portability and Accountability Act (HIPAA), participants were encouraged to be creative in their photographs in order to answer the prompts. Participants were provided nine weeks to take photographs and I also provided my contact information in case of any questions or concerns.

The prompts were broad and sought to capture snapshot of the lived experiences of CHWs (Table 2). The first prompt asked the participants to photograph an image that detailed what being a CHW means to them. The second prompt asked participants to capture an image of an impact they have had so far in their work. The final prompt asked the participants to document a challenge that they had overcome as a CHW. Participants were allowed to take as many photographs as they wanted but to submit only their top one or two images for each prompt for dis-

Table 1. Participant demographic characteristics.

\begin{tabular}{lc}
\hline Demographic characteristics & $\mathbf{N}=\mathbf{6}$ \\
\hline Age & 47 \\
Average & $20-65$ \\
Range & \\
\hline Sex & 2 \\
Male & 4 \\
Female & \\
\hline Race/Ethnicity & 3 \\
White & 3 \\
Latino/a & \\
\hline Years as community health worker & 5.93 years \\
Average & 3 months-30 years \\
Range & \\
\hline Level of education & 2 \\
High School Degree & 1 \\
Associate's Degree & 3 \\
Bachelor's Degree & \\
\hline
\end{tabular}

Table 2. Prompts and themes.

\begin{tabular}{lll}
\hline What does being a CHW mean to you? & What impact have you had as a CHW? & What is a challenge you have overcome as a CHW? \\
\hline - Educating clients & $\bullet$ Educating clients & $\bullet$ Becoming extroverted \\
\hline - Being present in the community & $\bullet$ Removing barriers to a healthier life & $\bullet$ Gaining a sense of self-confidence \\
\hline - Advocating for clients & $\bullet$ Aiding dying patients & $\bullet$ Paperwork/policies/laws \\
\hline - Empowering clients and community & $\bullet$ Navigating the health care system & $\bullet$ Overcoming idealized notions of the U.S. \\
\hline - Being nonjudgmental & $\bullet$ Providing access to care & $\bullet$ Finding resources \\
\hline Being observant & & $\bullet$ Navigating the large pool of resources \\
\hline - Not a duty, but an opportunity & & $\bullet$ Clients not qualifying for particular resources \\
\hline CHW, community health worker. &
\end{tabular}


cussion at the final presentation. The participants then gathered for a final meeting in which they discussed and interpreted their images.

\section{Analysis and documentation}

The second and final meeting was held nine weeks after the initial photovoice presentation and lasted two hours. The first hour of the meeting included a PowerPoint presentation that consisted of a compilation of the participants' photographs with the author serving only as the discussion facilitator. The photographs were shown first without the accompanying title and caption in order to evoke initial reactions from the other participants. This served as a crucial step in order to allow the other participants time to develop their own interpretation(s) of each photograph. The prompt was then included on-screen and the photographer read their caption, elaborated on their image, and how they felt the photograph served as a response to the prompt. Other participants were then given a chance to respond to the photograph and share their thoughts and interpretations. This process was repeated for each photograph and short discussion generally followed.

After every participant was given a chance to speak and share their photographs, they were asked a series of questions in order to further interpret, discuss, and summarize the photovoice experience during the second hour. Questions included whether or not the photographs and the accompanying captions were similar or different from their own experiences, which photograph(s) stood out the most, and if they would have done anything differently in terms of taking the photographs. One final question was posed to the CHWs, which asked how they would like to see the photographs used in the future. A lengthy discussion followed in which participants answered these questions. Following the conclusion of the second meeting, each participant was also took part in a 1-hour, semi-structured interview to further elaborate on his or her lived experience in relation to the overarching dissertation study. Several participants were also shadowed as part of data collection in the form of participant observation. As a point of clarification, the findings detailed in this study come directly from the photovoice project unless otherwise noted. Overall, participants had a total time commitment of four and half hours (between the photovoice meetings and semistructured interview) and varied in the amount of time they needed in order to capture the photographs.

\section{Results}

\section{Thematic analysis: general themes}

General and specific themes emerged through interpretation, analysis, and co-construction by the participants regarding their lived experiences. General themes were topics shared by a variety of the participants whereas specific themes were unique among each participant. How- ever, these interpretations were not solely offered by each individual participant but built upon and augmented through discussion as well. Below, I present the general and specific themes described by participants from the final meeting. In the Discussion section, I parse out some further applications and analyses from this project. Each prompt generated several main themes detailed in the caption of the photographs and through discussion (Table 2).

First, there was discussion regarding the general roles of the CHW position facilitated by the first prompt. Participants collectively identified being observant, serving as an advocate, being present in the community, and educating and empowering clients as the underlying meaning of being a CHW. Outreach in the community was a common theme throughout and was showcased by a majority of the photographs being taken outdoors. There was also lively discussion during the meeting in which participants described the importance of being nonjudgmental of clients and their living space. Rather than see negative aspects, participants described needing to look for the positives in their clients' life and home, especially as a motivating factor that might lead them to healthier choices and a sense of empowerment.

In terms of impact, participants identified a number of themes including health education, improving access to care, removing barriers to care, and navigating the health care system. Concepts such as opening doors, removing blindfolds, providing access were terms utilized in discussion around the impact of their work as CHWs. Navigating the health care system was an additional theme that fits within this context of improved access to care. Participants discussed that it was not enough to just provide access but also navigation through the complicated process of accessing health care, health insurance, diet change, and/or lifestyle change. Furthermore, participants emphasized that the end goal in this relationship to be client empowerment. One participant, who had received special training in performing palliative care assistance, added that her impact as a CHW revolved around being with patients who are dying. This was unique even among CHWs in that she has training to advocate and serve patients who are in the process of dying.

Lastly, the participants displayed photographs that demonstrated a challenge they have overcome as a CHW. Several of the shared surmounted challenges could be grouped into personal and work-related challenges. Two of the participants discussed how they had overcome personal challenges including a lack of self-confidence and learning to become extroverted. During the discussion, they noted how overcoming these personal challenges was crucial in order to improve their ability to advocate on behalf of their clients and in order to effectively conduct community outreach. In terms of work related challenges, participants described paperwork and operating within legal frameworks such as HIPAA. Although noting the importance of this law and various privacy policies, these produced challenges 
such as participants feeling as though they create a barrier in their relationship with their clients in addition to finding resources or advocating on behalf of clients.

Another common theme discussed was finding and acquiring health and social resources. While noting that Indianapolis has a large pool of resources to draw on participants explained the challenging aspect is that clients may or may not qualify for resources, some resources may only dry up due to running out of funding, being unaware that a particular resource exists, or that their client does not qualify due to legal status. Finally, the participants also discussed facing challenges in the form of whether or not clients were included within the health care system in addition to their own challenges of being included within the health care workforce. Below, I elaborate on several unique themes that emerged during analysis.

\section{Building individual character traits and skills}

Two of the participants noted how training and working as a CHW was vital in building their own character traits and abilities. Amanda [all names are pseudonyms], a CHW with only four months of experience, captioned her photograph of a turtle swimming in an aquarium as "a challenge I have had to overcome is not hiding in my shell and becoming more confident in myself". Adding to this concept of character growth, several participants also noted how the CHW certification course was vital in building skills and character traits crucial to the position in addition to learning how to be observant. This notion was encapsulated by one of Isabella's captions in which, to her, being a CHW meant someone who "must have big ears, big eyes, and a very, very small mouth." Although observation skills are an important facet of many medical professional jobs, participants asserted that CHWs spend more time with the clients and must listen and observe closely to truly understand the (health and social) issues in a client's life, identify proper resources, find ways to improve health, and, ultimately, empower clients. Participants especially noted the need to build character skills to learn about, advocate, and remove barriers for clients deemed noncompliant by medical professionals. Their discussion highlights the crucial ability for CHWs to address social determinants of health as many patients may be labeled as noncompliant yet are unable to adhere to a treatment plan due to transportation, financial burdens, time, or other structural factors. Finally, participants also utilized these new skills and enhanced character traits to seek out resources and advocate for their clients.

\section{An opportunity, not a duty}

A principal topic of discussion throughout the presentation consisted of the participants describing the primary function of their work as a CHW: as health educators, being present within the community, and advocating for their clients. Amanda described how being a CHW means "educating the people in our community, helping clients help themselves, and advocating for their needs." However, the worker portion of the CHW acronym might not adequately encapsulate the feelings of those who work in this position. This sentiment was expressed by Mark, a CHW with four months of experience, who answered all three prompts by creating a collage of photographs and writing a poem as his caption. Mark's poem described his collage in which he articulates that being a $\mathrm{CHW}$ provides him with an opportunity rather than simply a quotidian job (Figure 1). In extrapolating on this conception, Mark described how he utilized this opportunity to explore "dimly lit hallways" and to "open closed doors." He also outlined the possibilities of improving the health of the community through fostering new relationships and bringing hope into the lives of those most in need. Mark's comments provoked discussion among the participants about the uncertainties that occur in outreach but also the possibilities to truly improve the lives and health of individuals and communities.

\section{Putting the puzzle together}

Building off of the discussion regarding Mark's conception of the position, one participant described the importance of putting together seemingly unconnected pieces of a puzzle in order to find a solution a solution. Isabella, a CHW with four years of experience, displayed a photograph of a disconcerting scene she encountered one day while on a walk (Figure 2). Her photograph displays two boots, two toothbrushes, a day planner, and a tube of toothpaste strewn about a patch of grass. In answering how this photograph illustrated a challenge she has overcome as a CHW, she explained that an individual's life might seem complicated and that factors related to their lives may not seem to fit together. However, she emphasized the need to seek solutions and find commonalities between the different issues experienced by the client in order to effectively connect them to appropriate resources.

Isabella's comments provoked discussion among the participants who also described the how majority of their days are a puzzle in which they also must draw on their knowledge of the client's situation and available resources in order to provide effective aid. The participants, invoking her photograph, explained that helping a client might consist of a taking a variety of disparate resources and arranging them in a way to help their client. This was especially true as the participants described a wide availability of resources but that these were siloed. They described having to seek out various resources in their communities and/or had learned about resources that had not previously known about through word-of-mouth. Their discussion highlighted the essential component of CHWs serving as a knowledge base and resource connector for individuals and the community. 
Providing access and jumping into the U.S. health care system

A primary concern of the participants was the complexity of the U.S. health care system for their clients. Leticia, a CHW with more than thirty years of experience, displayed her photograph showing an individual at the banks of a large body of water. She visualized the U.S. health care system as a powerful river and pictured the client without knowing how to swim or possessing any tools to cross this treacherous water (Figure 3). Leticia described her impact as "helping patients know how to

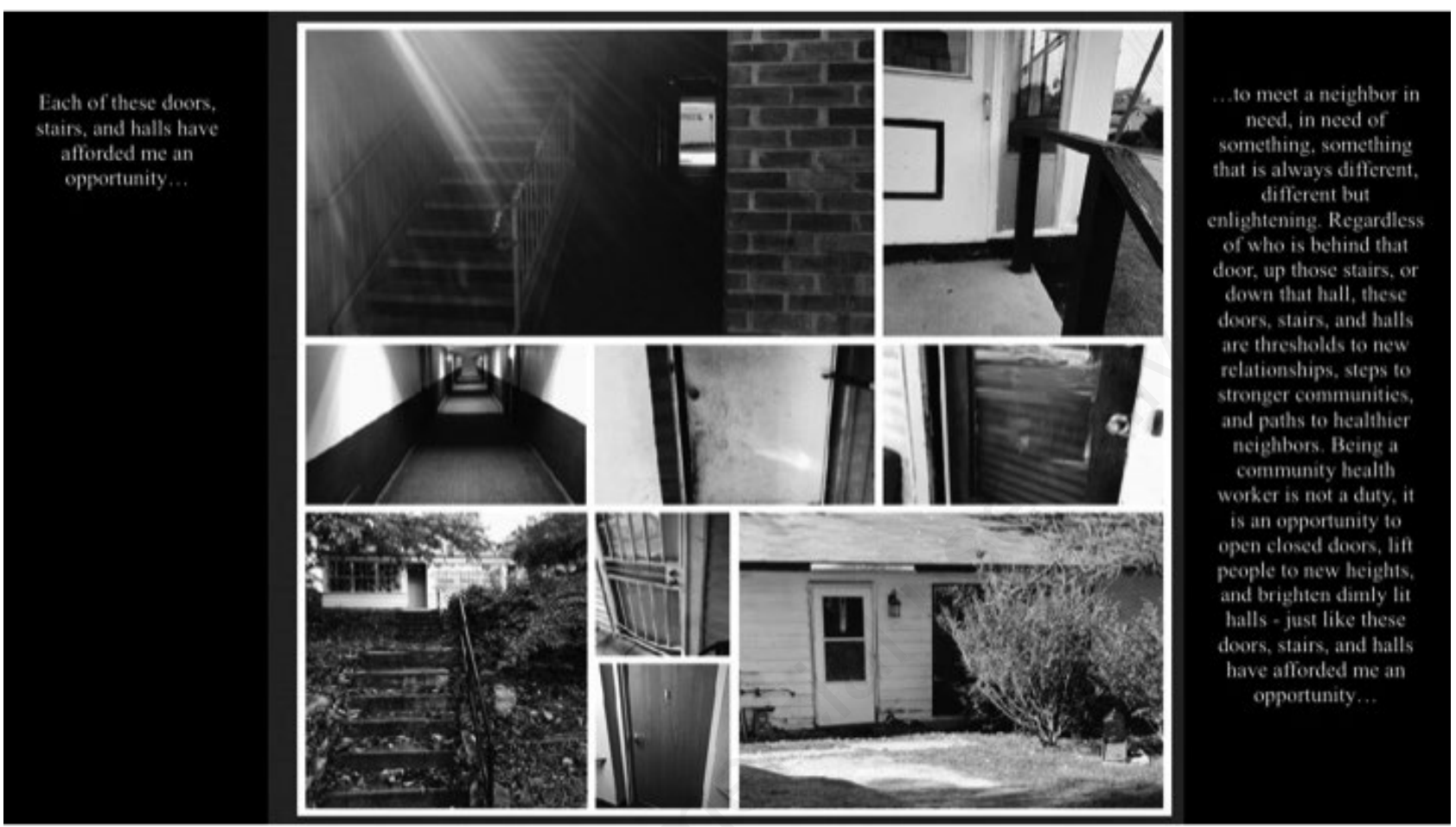

Figure 1. "Each of these doors, stairs, and halls have afforded me an opportunity...to meet a neighbor in need, in need of something, something that is always different, different but enlightening. Regardless of who is behind that door, up those stairs, or down that hall, these doors, stairs, and halls are thresholds to new relationships, steps to stronger communities, and paths to healthier neighbors. Being a community health worker is not a duty, it is an opportunity to open closed doors, lift people to new heights, and brighten dimly lit halls - just like these doors, stairs, and halls have afforded me an opportunity...".

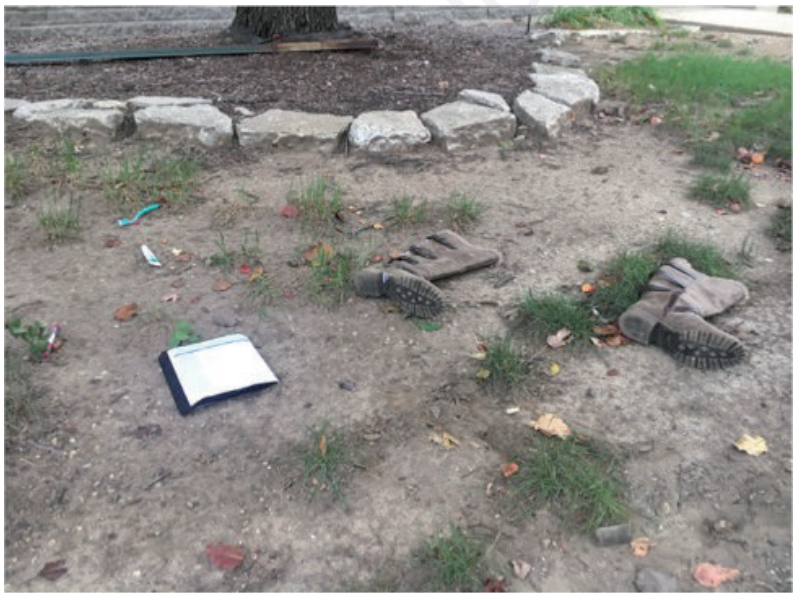

Figure 2. Prompt: What is a challenge you have overcome as a community health worker? "Many times, you may be apprehensive about what you see and doubt whether or not you have the ability to help put the puzzle pieces together."

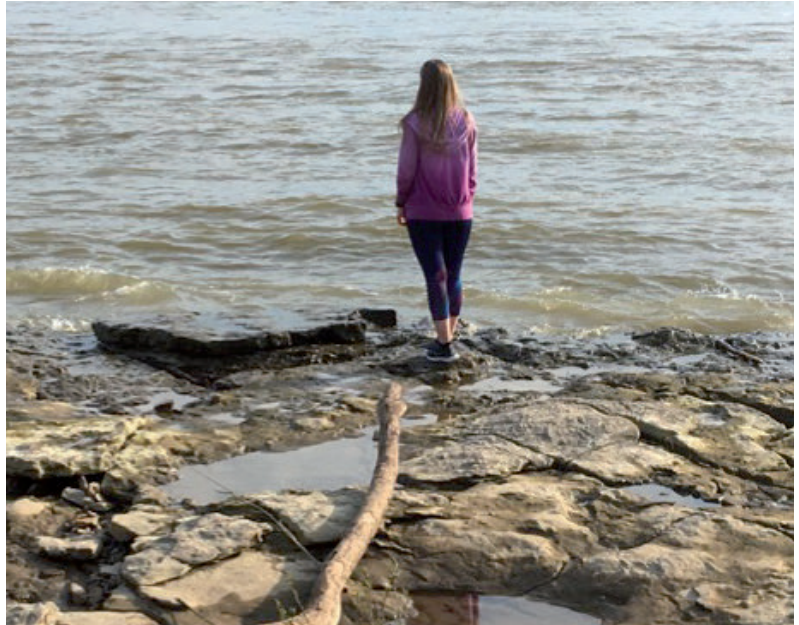

Figure 3. Prompt: What impact have you had as a community health worker? "Helping patients know how to jump into the health care system." 
jump into the health care system." The other participants discussed their critical role as providing access to care and educating clients on their options or where to go to seek specialized help.

However, participants stressed that it was not simply enough to provide access but to maintain support and navigation. They described helping clients explore health insurance options (and, if trained as an ACA navigator, help them sign up), calling clinics to schedule appointments with clients, going with clients to doctor's appointments, accessing public transportation, and providing ways to adjust diet and exercise. These services also underscored the need to teach and empower clients to take control once they had received help navigating the health care system and to take control of the next steps in their treatment plan. Leticia, who works predominantly within the Latino community, also emphasized specific challenges experienced by this population - especially for those who are undocumented. She described serving Latino immigrants in educating them on how to use the U.S. health care system since it was much different from the health care systems in their home countries. Other participants noted how other client populations, including low-income and homeless, also face similar challenges in accessing and receiving care. Ultimately, participants asserted that simply providing access was not enough and that continued support and navigation within the health care system was crucial.

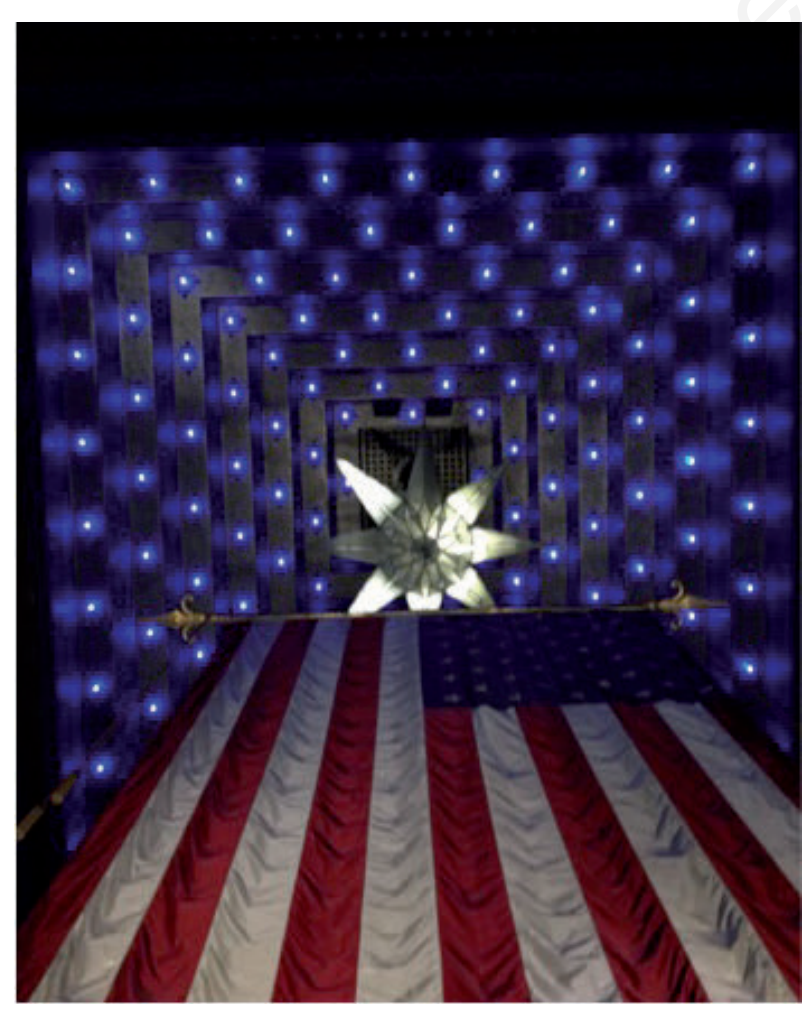

Figure 4. Prompt: What is a challenge you have overcome as a community health worker? "Overcoming the idea that the Land of Opportunity is for everyone."

\section{Overcoming idealized notions}

Following discussion of the difficulties that the most vulnerable populations encounter was discussion of how these issues contrast with idealized beliefs regarding the United States. This was specifically expressed by Leticia who described how the United States is idealized as the "land of plenty" yet there are significant portions of its population that require assistance. Leticia stated that she had to "overcome[ing] the idea that the Land of Opportunity is for everyone" (Figure 4). Participants found this image and description to be particularly profound yet unsurprising. As CHWs, the participants felt a connection to this image since they must be well aware of the available resources in their communities in order to connect clients to them. However, they discussed how clients from all different backgrounds may have trouble accessing these resources or were disqualified due to legal status, specific income requirement(s), and/or their geographic residence. This discussion of resources available in Indianapolis, the state, and in the U.S. also brought about topics relating to how the health care system is disjointed and difficult for clients to access. Moreover, extrapolating on how she has struggled to overcome viewing the U.S. as the "Land of Opportunity," Leticia related a story of the immigrant experience:

I mean there's that belief when they first arrive that this is the land of opportunity and they're going to help me and then you realize "well, you're not documented so we can't really help you." And when you have kids or people that are adults that are suicidal, while you can take them in for an assessment anywhere, you can do the assessment but then there's no treatment because they don't qualify. To me that's...it's hard to overcome that idea...

In pairing this story with her photovoice, Leticia provided a poignant vignette to other participants who did not work with immigrant populations - serving as one example of the empowerment education approach facilitated by photovoice. Despite these crestfallen sentiments, the participants still were motivated through their work and reiterated their perseverance to remain cognizant of the resources and empower clients to improve their lives.

\section{Community health worker recommendations}

A primary focus of the photovoice methodology is not only enhancing the voice of the participants but also including the participants' vision for how the data should be utilized. Following the presentation and discussion of the photographs, the participants were asked how they would like to see their photographs used. Mark expressed that he would like to see the photographs utilized in anyway possible that might help other CHWs and the position itself gain more awareness. Leticia suggested that the photographs be used in presentations given to medical professionals, potential employers, and policy makers. She further suggested having "lunch and learn" sessions in which members of the organization could showcase the 
position and use the photographs within the presentation. She stated "we show them these photographs and tell them from a medical perspective "what do you see behind those doors, or how would you find out what happened in this scenario?' And kind of begin to explain to them that a CHW may have an insight that is different." In this way, both issues of spreading awareness and demonstrating to employers how these workers can fit within their organization as an employee could be addressed. One of the participants, Dean, suggested creating a CHW calendar with these photographs. The calendar could serve as a fundraising item for the organization or could be distributed during presentations. The participants were also interested in having their photographs included on the organization's website along with a story about their photograph. In this way, participants were keen on making an applied impact from their participation in this project.

\section{Discussion}

Photovoice served as a method that illuminated the lived experiences of CHWs. As other studies quantitatively demonstrate the how these workers can impact the health of clients, ${ }^{16}$ photovoice reveals the nuances and intimate details at the grass-roots level. Several studies have previously utilized photovoice to explore a variety of topics with these workers. ${ }^{13-15}$ These studies demonstrate that photovoice can be used to effectively showcase, understand, and analyze these issues and can also be used to produce data that fosters critical consciousness and to provoke social change. Given that individuals who may be unaware of the complex landscape navigated by CHWs often craft policy regarding these workers, further understanding of their unique experiences is vital when developing policy. This method also provides an outlet for CHWs to illuminate personal experiences, showcase these to their peers, co-construct meaning, and put their findings into action.

The themes that emerged during this project also contribute to current conversations on the topic of CHWs. In their report on the voice of CHWs, citizens' right to health, and power, Schaaf et al..$^{22}$ explore issues and challenges impacting these workers in the U.S. and abroad. ${ }^{22}$ Particularly, Schaaf et al..$^{22}$ explore the theme of accountability and assert that CHWs are well placed to be agents of downward accountability, that is, assuring fulfilling the accountability of the health system to all people. ${ }^{22}$ As with CHWs throughout the world, the participants in this study drew on their connections to their communities in order to promote health and social wellbeing. Furthermore, participants noted working with a variety of organizations and stakeholders in the pursuit of improving the lives of the clients. They note the variety of actors - including the state and other stakeholders (community, NGOs, other organizations) - that impact the roles and utilization of CHWs. ${ }^{22}$ These authors state that understanding CHW accountability to stakeholders and community is vital. Ad- ditionally, understanding the barriers and issues on the ground will help employers empower their CHW employees who can then more effectively serve their communities and clients. Emergent themes from this project highlight the downward accountability that participants had to their communities in addition to seeking ways to facilitate the provision of resources to their clients.

This photovoice project also provided participants with an opportunity to explore several themes related to their lived experiences. Policy makers, researchers, and other stakeholders must be cognizant of the lived experiences of CHWs when implementing interventions or developing policy. Since the state government of Indiana has already begun work to integrate CHWs into the broader workforce, the findings from this project can be used to reach a wider audience in order to demonstrate the utility of these workers throughout the state. The photovoice prompts, although broadly related the successes, challenges, and impacts, provided a creative means for participants to answer them. As a result, the participants in this project provided unique insights to their character development and key roles of this underutilized workforce fulfill within their communities. The photographs and findings from the discussions can be used in upcoming presentations with stakeholders including policy makers and potential employers as a means to demonstrate the responsibilities as CHW can perform if employed. Finally, participants identified specific challenges for policy makers and stakeholders to consider including disjointed resources, issues in the U.S. health care system, whether or not clients qualify for resources, and strategies to solving these complicated situations.

\section{Legitimacy of community health workers in the workforce}

In Indiana, where CHWs continue to operate on the fringes of the health care workforce, educating stakeholders is crucial for enhancing their integration within the formal the health care environment. Medical citizenship is a theoretical lens that describes the belongingness of a group or individual(s) within a health care system. ${ }^{23,24}$ This also includes conceptions of who is deemed deserving of care, including those who have access to health resources. ${ }^{23,25,26}$ This is a useful framework to assess not only the inclusion and/or exclusion of CHWs within the health care system but also the communities they serve as well. Nading ${ }^{24} \mathrm{em}-$ ployed medical citizenship in his research with brigadistas, a term for CHWs in Nicaragua. He argues that brigadistas are at once professionals that instruct community members in ideal, healthy behaviors while at the same time serve as caring advocates for their communities. In exploring this duality of belonging, Nading (pp. 97-98) 24 asserts that brigadistas must "balance two contradictory forms of medical citizenship, one disciplinary and bureaucratic, the other compassionate and flexible."

This is true of CHWs in Indiana as they navigate be- 
tween citizenship within their communities and the professional workforce. Leticia's photographs (Figures 3 and 4) and vignette specifically highlighted the lack of inclusion faced by her clients within the U.S. health care system. In the semi-structured interviews, participants noted how the communities they work within - especially immigrant communities - were restricted from medical care and thus deemed undeserving and without medical citizenship. Additionally, for CHWs themselves, lacking medical citizenship within the professional community could stymie the provision of care and resources provided by these workers.

Other scholars have described how CHWs encounter this tension within their work environment. ${ }^{27,28}$ These scholars describe how these workers are in a position in which they must advocate not only for their community but for their job as well. ${ }^{27,28}$ Moreover, as CHWs are typically members of their communities, they face the same structural issues as their clients. ${ }^{22,27,28}$ Sabo et al.$^{28}$ argue that the same structural inequality that hinders the individuals in their community from upward social mobility also impedes professional mobility for these workers. In this way, CHWs are only partly belonging within the health care workforce and share many of the same structural vulnerabilities as their community members. Additionally, several participants described in the semi-structured interviews as being seen with suspicion and apprehension by medical professionals thereby underscoring their lack of medical citizenship within this workforce. This highlights the precarity of the dual medical citizenship navigated by CHWs in Indiana - much as described by Nading. ${ }^{24}$

Although there has been ostensible federal support for the inclusion of CHWs, the position itself is typically excluded as an additional member of the health care team. ${ }^{12}$ In spite of this, other scholars have noted the potential for CHWs to be included as a distinct member of the health care team. ${ }^{5,16}$ Acquiring formal inclusion within the workforce is especially important as CHWs in Indiana are funded through soft-money and their positions wax and wane depending on grant funding. This photovoice project was vital in demonstrating the nuances of the roles fulfilled by CHWs and showcasing their lived experience.

\section{Community health worker belongingness and client deservingness within the nation-state}

Leticia's photograph (Figure 4) showcases how she now views popular conceptions regarding the United States in a more nuanced manner. This is true to of how her clients, who are mostly Latino immigrants, have to reconcile with the fact that opportunity and health services may be relatively cut off from them. While the CHWs in this sample served a diverse range of racial and ethnic backgrounds, understanding the relationship of these workers to the nation-state is a crucial finding of this study. This lack of belongingness was seen in Leticia's photograph about the United States, which left her and her broader community feeling excluded from the health care system.
Leticia's comments and discussion relating to Latino health reiterated the difficulties of both documented and undocumented immigrants in the U.S. health care system - especially as scholars have argued that immigration itself be viewed as a social determinant of health. ${ }^{29,30}$ The concept of health-related deservingness is useful and can be described as the moral, economic, and political sentiments that mandate whether or not a population is worthy of care. ${ }^{25,26}$ Language barriers, legal status, financial barriers, and political sentiments have and continue to mark immigrant populations as undeserving of care. These concepts can also be applied to other minority groups, low-income, and/or homeless populations who also faced politicized notions about their deservingness of health resources. Leticia's photograph and the resulting discussion again invoke the vulnerabilities faced by the communities served by CHWs in this project yet also the potential for advocacy and health education as a means to circumvent these issues.

\section{Increasing access to resource and health services}

Several of the emergent themes from this project, including putting the puzzle pieces together and providing access to care and jumping into the U.S. health care system, demonstrate how participants navigate the complex economy of resources within Indiana in order to provide access to health and social services. Participants described empowering their clients and communities as a key part of their work. As members of their respective communities, these workers have an intimate knowledge of the structural barriers and health disparities of their clients. CHWs connect their clients to resources, educate clients on a variety of topics, and advocate and empower clients to take control of their health. Participants in this project also noted the variety of resources available yet lack of awareness or education on part of the client hindered their usage. Participants also noted how they served to advocate for their clients as well as empower them to overcome social determinants of health. Addressing the social determinants of health is a vital step toward breaking down health disparities and leading toward improved health equity. Finally, steps to integrate these workers must include establishing permanent positions in the workforce rather than continuing to employ them through soft-money. This will serve as a means to increase the amount of stable CHW jobs.

\section{Community health workers' understandings of their position}

Another unique insight from this project relates to how the participants described their own conception of the position and how undergoing the certification course impacted their personal character. Mark's notion of conceptualizing the CHW position as an opportunity rather than a duty underscores his deep and personal impetus behind his reasoning for participating in this job. Other participants described how the certification course 
pushed them to further develop their own personal character traits. This was noted through character traits identified in the course as being extroverted and possessing a willingness to advocate for clients and the community. This part of their lived experience is essential to highlight as it inserts the voice of the CHW into the literature and discussion regarding the development and direction of their position. Previous scholarship has documented the potential for this position to lose its unique contributions by becoming over-medicalized, narrowly defining the roles of the position, and/or not including CHWs in discussions regarding its development. ${ }^{12}$ Thus, as legislators and stakeholders continue to write policy and approve certification, understanding how these workers view their position and how, in turn, the position shapes their personal characteristics is vital in retaining the essence of the work when developing policy.

\section{Community health workers as natural researchers}

This project also highlighted the roles that CHWs can play in conducting projects with academic researchers. As noted in the literature, these workers have the potential to contribute to studies and research carried out in conjunction with health researchers. ${ }^{10-12}$ Pérez and Martinez ${ }^{12}$ label CHWs as natural researchers due to their close connection with their community and their ability to suggest solutions to address community issues. These close connections offered by CHWs have the potential to be collaborators on health programs and can enhance the effectiveness of these programs. Pérez and Martinez (p. $11)^{12}$ argue that these workers "can observe and relay community realities to outsiders - placing them in a position to influence policy that affect public health.” The participants in this project demonstrated their close connection to their communities in addition to their knowledge related to the health issues, social determinants of health, and resources available. As a result, these workers have the potential to dramatically affect health programs, policy, and research. Additionally, the governor's workgroup in Indiana has included "evaluation and research skills" as a core competency of CHWs, which asserts their ability to aid in research projects. This further strengthens their potential role in research and policy development.

\section{Implications for potential employers and integration of community health workers}

There are several implications that arise from this project in documenting the lived experiences of CHWs. As public health scholarship has quantitatively demonstrated positive health outcomes utilizing $\mathrm{CHW}$ interventions as well as cost-effectiveness, ${ }^{16,18}$ these workers may have significant impacts on the health care landscape of the U.S. Leticia, Mark, and Isabella specifically demonstrated their roles as connectors between health/social services and their clients as well as their extensive outreach (Figures 1-3). By taking into account their lived experiences, public health programs can better tailor health interventions and outreach initiatives. In addition, learning about the specific issues that CHWs encounter on the ground and how they have successfully navigated these challenges will better inform these programs. However, it is crucial to note that these workers should not be seen as a single solution to attaining health equity. Colvin and Swartz ${ }^{29}$ assert that CHWs should be supported internally but should not shoulder the burden of fixing a broken health system. Instead, CHWs can improve access to care, reduce cost and readmission, and improve health equity for their clients. ${ }^{29}$ Overarching systemic change must come from elsewhere, with CHWs as supporting actors.

\section{Limitations and future research}

There are several limitations to this research project. While the sample size was small $(n=6)$, it touched on some prevalent issues related to the lived experiences of CHWs in Indiana. Although the original sample size was eight individuals, two participants were lost to followup after the initial meeting due to time constraints. Wang ${ }^{8}$ recommends between 7-10 participants for a photovoice project. Due to the small sample size, data was augmented through the use of semi-structured interviews and participant observation to further elaborate on the themes discussed in the photovoice project. Aside from the small sample, recruitment was also difficult. CHWs work within the same legal boundaries as medical professionals and thus taking photographs may have posed too great a difficulty for some. Also, the time constraints of the participants made it difficult to meet more than twice. Additional meetings would have allowed for a deeper analysis of the photographs and experiences of the participants.

Moreover, additional prompts may help fill in gaps regarding negative experiences encountered by CHWs. Although the majority in this study described positive experiences, larger sample sizes and at least one prompt about negative, challenges, or criticisms may help parse out additional nuances regarding the lived experiences of CHWs. Another limitation included that, at the time of the publication, the organization was still developing presentations and materials from the findings of this research. Given the organization's small staff and busy work schedules, implementing the findings through the recommendations remains to be seen.

A final limitation (and consideration for future research) should assess the power dynamics between CHWs and their clients. The U.S. health care system and by extension, CHWs, are impacted by neoliberalism and its impact on health and individual responsibility. While these workers seek to empower their clients through an emphasis on eventual self-sufficiency, these workers also attempt to manipulate and change the system to the benefit of their clients through participation in advocacy. An ex- 
ploration of the power dynamics between CHWs and clients was not specifically explored throughout the context of this project but future studies should further assess this relationship and the impact of the neoliberalism on the CHW model.

Future research should arrange a photovoice project with CHWs tackling a particular health issue. These future studies might examine the health impact of $\mathrm{CHW}$ interventions aiming to reduce $\mathrm{A} 1 \mathrm{c}$ levels, enhance nutrition, or an exercise program with a particular population. These studies could also conduct a joint $\mathrm{CHW} / \mathrm{client}$ photovoice project to fully explore relevant issues related to these programs. Lastly, future studies should consider recruiting CHWs who all work in the same agency as a means to improve loss-to-follow up as they will be centrally located and could more easily attend meetings and continue the project to completion.

\section{Conclusions}

This project explored the nuances of the lives and work of CHWs in Indiana through the method of photovoice. Specifically, their photographs served as a lens to explore how participants conceptualized their work, the challenges they have overcome, and the impact they have had on their communities. This method and, specifically, its participatory approach and theoretical framing, serves as a mechanism to elevate the voice of the participants the project and foster critical consciousness. Through the sharing of photographs and interpretations, participants co-constructed their lived experiences as CHWs. As a community themselves, participants found meaning not only in the lived experiences but also through offering suggestions for how these findings can potentially improve their communities and professional livelihoods. Ultimately, the participants and their findings offer not only insight into their lived experiences and unique contributions to the health care landscape but also data that can generate social change.

\section{References}

1. Bureau of Labor Statistics. Occupational employment and wages, May 2017. 21-1094 Community health workers. 2017. Available from https://www.bls.gov/oes/current/oes 211094.htm

2. Ingram M, Reinschmidt KM, Schachter KA, et al. Establishing a professional profile of community health workers: Results from a national study of roles, activities and training. J Community Health 2012;37:529-37.

3. Rosenthal EL, Brownstein JL, Rush CH, et al. Community health workers: part of the solution. Health Aff 2010;29: 1338-42.

4. Najafizada AM, Bourgeault IL, Labonte R, et al. Community health workers in Canada and other high-income countries: A scoping review and research gaps. Can J Public Health 2015;106:e157-64.

5. Balcazar HG, Rosenthal EL, Brownstein JN, et al. Commu- nity health workers can be a public health force for change in the United States: Three actions for a new paradigm. Am J Public Health 2011;101:2199-203.

6. London K, Damio G, Ferrazo M, et al. Addressing social determinants of health through community health workers: A call to action. Policy brief. Hispanic Health Council. 2018. Available from http://hispanichealthcouncil.org/images/ Brief2018.pdf

7. Kangovi S, Grande D, Trinh-Shevrin C. From rhetoric to reality - community health workers in post-reform U.S. health care. New Engl J Med 2015;372:2277-9.

8. Wang C. Photovoice: A participatory action research strategy applied to women's health. J Women's Health 1999;8:185-92.

9. Wang C, Burris MA. Photovoice: Concept, methodology, and use for participatory needs assessment. Health Educ Behav 1997;24:369-87.

10. Johnson CM, Sharkey JR, Dean WR, et al. Promotoras as research partners to engage health disparity communities. J Acad Nutr Diet 2013;113:638-42.

11. Nebeker C, Kalichman M, Talavera A, Elder J. Training in research ethics and standards for community health workers and promotores engaged in Latino health research. Hastings Center Report 2015;45:20-7.

12. Pérez LM, Martinez J. Community health workers: Social justice and policy advocates for community health and wellbeing. Am J Public Health 2008;98:11-4.

13. Baquero B, Goldman SN, Simán F, et al. Mi cuerpo, nuestro responsabilidad: Using photovoice to describe the assets and barriers to sexual and reproductive health among Latinos. J Health Dispar Res Pract 2014;7:65-83.

14. Mayfield-Johnson S, Rachal JR, Butler J. When we learn better, we do better: Describing changes in empowerment through PhotoVoice among community health advisors in a breast and cervical cancer health promotion program in Mississippi and Alabama. Adult Educ Q 2014;64:91-109.

15. Mitchell C, DeLange N, Moletsane R, et al. Giving a face to HIV and AIDS: on the uses of photo-voice by teachers and community health care workers working with youth in rural South Africa. Qual Res Psychol 2005;2:257-70.

16. Allen JK, Himmelfarb CR, Szanton SL, Frick KD. Cost-effectiveness of nurse practitioner/community health worker care to reduce cardiovascular health disparities. J Cardiovasc Nur 2014;29:308-14.

17. Arvey SR, Fernandez ME, LaRue DM, Bartholomew LK. When promotoras and technology meet: A qualitative analysis of promotoras' use of small media to increase cancer screening among South Texas Latinos. Health Educ Behav 2012;39:352-63.

18. Cross-Barnet C, Ruiz S, Skillman M, et al. Higher quality at lower cost: Community health worker interventions in the health care innovation awards. J Health Dispar Res Pract 2018;11:150-64.

19. Carlson ED, Engebretson J, Chamberlain RM. Photovoice as a social process of critical consciousness. Qual Health Res 2006;16:836-52.

20. Langhout RD. Photovoice as methodology In: Schenker MB, Castañeda X, Rodriguez-Lainz A, eds. Migration and health: a research methods handbook. Oakland: University of California Press; 2014. pp 327-342.

21. Bade B, Martinez K. Full circle: The method of collaborative anthropology for regional and transnational research. In: Schenker MB, Castañeda X, Rodriguez-Lainz A, eds. 
Migration and health: a research methods handbook. Oakland: University of California Press; 2014. pp 306-326.

22. Schaaf M, Warthin C, Manning A, Topp S. Report on the 'think-in' on community health worker voice, power, and citizens' right to health. 2018. Available from http://accountabilityresearch.org/web/wp-content/uploads/2018/01/Learning Exchange Report3 January-18-1.19.18.pdf

23. Nichter M. Global health: why cultural perceptions, social representations, and biopolitics matter. Tucson: University of Arizona Press; 2008.

24. Nading A. Love isn't there in your stomach: a moral economy of medical citizenship among Nicaraguan community health workers. Med Anthropol Q 2013;27:84-102.

25. Goldade K. Health is hard here or health for all? The politics of blame, gender, and health care for undocumented Nicaraguan immigrants in Costa Rica. Med Anthropol Q 2009;23:483-503.
26. Horton S. Different subjects: The health care system's participation in the differential construction of the cultural citizenship of Cuban refugees and Mexican immigrants. Med Anthropol Q 2004;18:472-89.

27. Closser S. Pakistan's lady health worker labor movement and the moral economy of heroism. Ann Anthropol Prac 2015;39:16-28.

28. Sabo S, Wennerstrom A, Phillips D, et al. Community health worker professional advocacy. J Ambul Care Manage 2015;38:225-35.

29. Colvin CJ, Swartz A. Extension agents or agents of change? Community health workers and the politics of care work in Postapartheid South Africa. Ann Anthropol Prac 2015;39: 29-41.

30. Castañeda H, Holmes SM, Madrigal DS, et al. Immigration as a social determinant of health. Annu Rev Public Health 2015;36:375-92. 